\title{
Digital Authoring of Interactive Public Display Applications
}

\author{
Ryan Mills \\ Lancaster University \\ Lancaster, England \\ r.mills2@lancs.ac.uk
}

\author{
Matthew Broadbent \\ Lancaster University \\ Lancaster, England \\ m.broadbent@lancs.ac.uk
}

\author{
Nicholas Race \\ Lancaster University \\ Lancaster, England \\ n.race@lancs.ac.uk
}

\begin{abstract}
HbbTV (Hybrid broadcast broadband TV) is an emerging force in the entertainment industry, and proper standarisation of technologies would be hugely beneficial for the creation of content. HbbTV aims to realise this vision and has been widely successful thus far. This paper introduces the MPAT (Multi Platform Application Toolkit) project, which is the result of multiple organisational entities effort and dedication to extend the capabilities and functionality of HbbTV, in order to ease the design and creation of interactive TV applications. The paper also showcases the versatility of MPAT, by describing a series of case studies which provide digital storytelling and visual authoring of interactive applications which transcend traditional TV use cases, and instead provide a gripping interactive experience via integration with public displays.
\end{abstract}

\section{Author Keywords}

Public Display Infrastructure; Interactive TV Applications; Authoring Tool

\section{CCS Concepts}

-Human-centered computing $\rightarrow$ User interface management systems;

\section{INTRODUCTION}

Interactive TV applications require a lengthy process of authoring, reviewing and publishing which remains expensive with significant upfront investment typically needed. Furthermore, expertise in this technical field also requires trained specialists capable of implementing bespoke complex architectures. In addition to the lengthy review process, applications naturally take extensive time before they are fit for deployment. MPAT [13] is described in [15] as "an open-source authoring and publishing tool, based on the WordPress content management system and provides an easy-to use solution to existing publishing systems that supports rapid expert review and professional online publishing". Both WordPress and MPAT are open source projects, and the latter extends the functionality of WordPress to enable the creation of powerful interactive TV applications. The use of WordPress as a base Content Management System (CMS) has various advantages over a bespoke system designed to achieve the same goal, namely that it is a

Permission to make digital or hard copies of all or part of this work for personal or classroom use is granted without fee provided that copies are not made or distributed for profit or commercial advantage and that copies bear this notice and the full citation on the first page. Copyrights for components of this work owned by others than the author(s) must be honored. Abstracting with credit is permitted. To copy otherwise, or republish, to post on servers or to redistribute to lists, requires prior specific permission and/or a fee. Request permissions from permissions@acm.org.

CHI'16, May 07-12, 2016, San Jose, CA, USA

(C) 2016 Copyright held by the owner/author(s). Publication rights licensed to ACM. ISBN 123-4567-24-567/08/06...\$15.00

DOI: http://dx.doi.org/10.475/123_4 hugely popular tool, with a dominant market share compared to other CMS options [6]. This aspect alone presents a strong argument in favour of the use of this system, as there is a substantial likelihood that users will already have familiarity in the creation of similar pages. Similarly, Wordpress is robustly tested, and has a vibrant community who continually build additional functionality. As a visual alternative to the creation of web pages, WordPress has been demonstrably shown to be easy to learn and is ideal for simple web pages. This simplicity is advantageous when designing TV applications as complexity and verbosity will often confuse the end-user. MPAT aims to enable the creation of, otherwise very technical and expensive, TV applications by users who lack the expertise to do so. This is done in an attempt to increase the number of applications for TV, and reduce the time taken.

During the design, testing and implementation of MPAT, it became clear that it would support a myriad of potential use cases outside of the TV traditional broadcast settings. This is in part due to the nature of the application created; simple and intuitive in use. The applications that MPAT creates are HbbTV compliant; a standard adopted by manufacturers to ensure cross-compatibility of TV applications. Current uses of this application include additional and interactive content, which complement a relevant broadcast TV programme. However, due to the power and ease of use that this visual editor provides, it is feasible for use outside of the TV application domain. There exists similarities between the way in which interactive TV and public display applications are consumed, including the size of the displays, the necessity to display information clearly and concisely, and the distance between the display and the viewer. The interaction techniques are also similar: operated by one person whilst having multiple viewers. Furthermore, both public displays and TV share components within their problem domain. The design of public display applications is inherently difficult due to the careful choices needed to ensure engagement, while TV applications must subtly, yet effectively, imply the notion that an application is available for execution whilst the broadcast is in the background. Moreover, the creation of said applications is demanding in itself as these displays often operate on a diverse range of hardware, and the application must be implemented using disparate guidelines depending upon the ownership. This issue has been recently remediated through the standardisation of HbbTV technology for TV environments, yet no such standard exists for public displays. As MPAT offers simultaneous availability for multiple platforms, whilst also streamlining the creation process, it becomes a viable candidate for creating public display applications. 


\section{PUBLIC DISPLAY INFRASTRUCTURE}

The context for the development of MPAT for public displays was Lancaster University, which hosts a large-scale PDI (Public Display Infrastructure) [14]. This infrastructure consists of a number of connected displays that are capable of presenting personalised content to each individual user, which is achieved by an integration of iBeacon technology. This effort to personalise content on the various displays allows for more engaging content, and allows students the means to find specific information on demand. The current implementation requires use of an $\mathrm{iOS}$ application developed by researchers at Lancaster University: 'Tacita' [12].

The technology underpinning this has also been merged with the hugely popular 'iLancaster' application, which is available for both Android and Apple devices and is installed by the majority of Lancaster University students and staff. Tacita aids developers in the creation of custom applications which communicate with the PDI to display personalised content on individual screens across campus when a user has come into contact with them. If enabled, Tacita will periodically check to see if a user is in the vicinity of a public display that provides applications matching the users preferences. If so, the mobile application contacts the relevant web-based display application, specifying the desired display and personalisation parameters. The web application then contacts the display component in order to ensure that the relevant content was shown on the display.

Mobile devices which act as interaction gateways may alleviate problems relating to engagement and interaction inherent within public displays. These displays often do not give the feeling of ownership to their users in the same way that a personal device does, which in turn imposes difficulties for user engagement [5]. In comparison to other interaction procedures such as touch enabled interactions, mobile devices grant the user a certain level of privacy and familiarity.

In this paper, we aim to design and implement a number of public display applications using MPAT. Two distinct use cases are investigated, as we highlight both the build and deployment processes for each. Finally, we explore the interaction possibilities, as well as conducting a preliminary evaluation of the use of these applications.

At present, it is believed that no applications using HbbTV's library and specifications have been adopted for use by public displays. As a result, research into this area is very scarce and careful consideration of the implications that this transition creates must be taken into account when designing such applications. At MPAT's core lies a fundamental objective to allow its service to be be available on multiple platforms. This paper outlines the design and interaction procedure relating to this process.

\section{RELATED WORK}

There exists a large amount of tools which aim to reduce the time spent designing interfaces for applications. However specialised applications, such as ones applicable to interactive TV and PDIs, are less well known. While the HbbTV foundations are built using accepted standards, they require some expertise in order to properly extend their functionality. Nevertheless, tools of this nature have been developed, and are documented below.

Other products exist that compete with MPAT, which all aim to extend upon the HbbTV library and provide a comprehensive visual authoring environment. HEd [8] is a WYSIWYG (What You See Is What You Get) visual authoring tool which facilitates the creation of interactive TV applications in a similar manner to MPAT. Generic components can be placed at will within a visual interface, representing the application within a broadcast stream upon rendering on a display. This variant provides an interface for the customisation of displayable elements by the allowing an author to specify the CSS properties that they desire. These components can also be modified to include custom functionality that a specific application requires by the utilisation of JavaScript (JS). functions. While services such as these exist and provide much of the functionality present within MPAT, none of them been implemented within the sphere of public displays.

There has been substantial research into pervasive display design patterns, which aim to successfully draw attention and engage with viewers to ultimately begin the interaction procedure. Bendinelli [7] identifies many of these patterns through the evaluation of public display utilisation. These patterns outline certain recurring situations and provide insight into how to address them, while also providing a starting point for the application development process. While a useful starting point, these guidelines must be extended in order to provide a meaningful experience for users upon consideration of a specific application. A development environment which realises these guidelines was also created. This environment aims to highlight the most important aspects that compose the structure of such applications, by allowing an author to exploit a list of patterns and apply them to their application. As this environment does not allow for visual arrangement and addition of components, the development process can be seen as less intuitive in comparison to the editor provided by MPAT. While MPAT does not provide guidelines for development, many high level components are packaged with MPAT, and an author is free to experiment with many possible design scenarios. These components are also readily customisable within the MPAT editor, and also extensible by the modularity which WordPress provides.

The choice of a web based framework to produce interactive public display applications is not an uncommon approach, as this method is at a high level of abstraction and allows content to be shared from a number of sources. These applications conform to common web technology standards such as HTML and JS. An example of this is the UBI hotspots, which is a pervasive display infrastructure which bases its design on the web paradigm. This abstraction grants services which reside on the Internet and accessed using a URL to be consumed by the various public display deployments situated in Oulu, Finland [9]. Akin to the deployment lead by Lancaster University, users can initiate interaction using personal mobile devices.

Although the number of public displays is large and continues to grow, there exists a small percentage which can actually 
be interacted with. Cardoso states that this is due to the fact that there exists no clear abstraction for interaction modality, and this leads to specific work, outside of the core application functionality, having to be undertaken and replicated by each developer working on a separate project [10]. With this lack of abstraction impacting the quality of public display applications, a clear standard implementation for such systems would be beneficial. Cardoso also creates an environment which allows developers to ignore the lower level details of interaction implementation, and instead focus on core functionality. Although this product exists, it is very bespoke and doesn't offer a wide range of functionality. MPAT offers its services on multiple platforms and its base, HbbTV, has been rigorously tested by industry leading organisations as it is not a drastically new implementation. This mature technology provides an open API for interaction, and could be a clear contender for the standard abstraction service for public displays.

\section{DESIGN}

As part of an ongoing initiative to improve HbbTV adoption across the UK, Lancaster University have conducted two very different pilot projects. Each of these aims to draw attention to the emerging technology while providing end-users the opportunity to interact with MPAT enabled HbbTV applications.

\section{Overview of Pilots}

The pilots demonstrated and documented in this paper consist of one which caters to prospective students and one for the general collegiate. The former is focused on building an electronic prospectus for students visiting Lancaster University on one of our open days. The latter integrates with the existing PDI lead by Lancaster University, to create a storytelling narrative for staff and students. They will receive a piece of content, visible on the multiple displays located around campus as they are encountered. For the purposes of this paper the former pilot will be referenced as the digital prospectus pilot, and the latter will be referenced as the presence detection pilot.

The presence detection pilot follows the interior lives of three characters: The Scientist, The Artist and The Historian until the egress of a celestial event overlooking Bowland Tower within Lancaster University. The narrative is distributed and non-linear in nature, allowing users to explore the various possible story lines possible. The story will evolve depending upon the order of displays visited by, in essence this means that each individual user will explore a different narrative across campus. A visual representation of this is depicted in Figure 1. In the example, each display is attributed to one of the three story lines, meaning that when a user encounters a display, they will receive a video depending upon how far into the story they are and also which story line the display is connected to. The overall story has five separate instances which users can experience, with the ability to see parts from each of the three overarching character narratives, dependent upon which displays the user encounters around campus. While each character has it's own narrative, the content within each storyline seamlessly flow into one another to create a unique experience encompassing the different thematic representations a celestial event.
Figure 1. The three narratives of the storytelling application

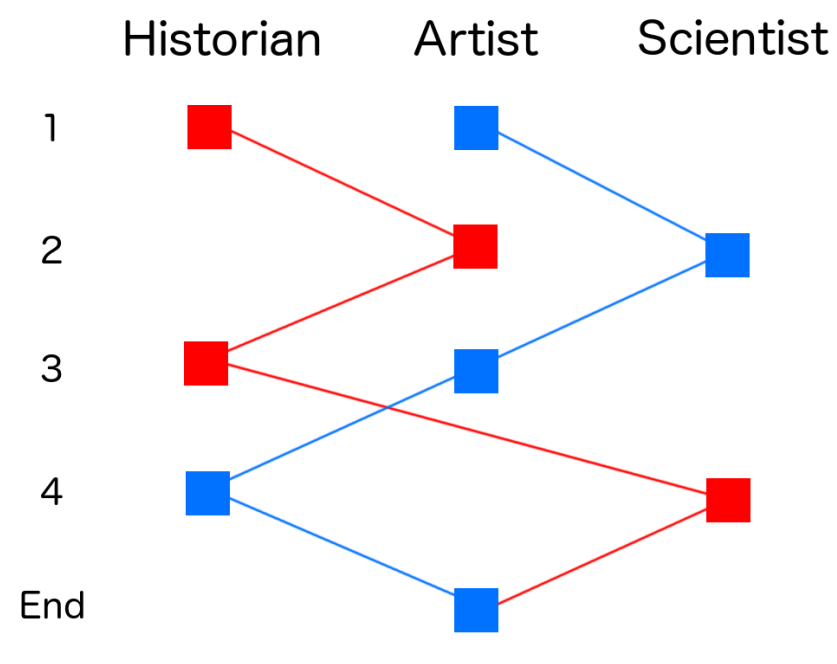

The digital prospectus pilot, while deployed in a public location, does not integrate with the display infrastructure Lancaster University has developed. The application is launched on a standard digital TV, with a traditional broadcast stream being produced by a connected computer, which provides a looping informational video. This pilot showcases MPAT in its traditional format, while also being in a public setting. Because of the fact that the application is not broadcast with terrestrial TV and is not to be consumed in a home environment, several choices in design must be made that accommodate this.

Firstly, as it is a public settings and there are multiple stimuli in close surroundings, it must be easy to identify that the display exists and is providing information, and furthermore that there is an additional application which exists and can be interacted with. The display must be situated in such a manner to catch the eye of prospective students as they enter and also maneuver around the various attractions on offer by the University. The method of input must also be made easily accessible and intuitive to use, and care must be taken to increase engagement which also neither obstructs or is obtrusive to other potential interacting users [5]. The latter pilot is centered around integrating the existing PDI offered by Lancaster University with MPAT. Research into correct substantiation of content for viewing within pervasive display infrastructures is vast and encompasses multiple view points. Linden et al [2] propose a web-based framework to cater the process of content instantiation and interaction. The paper describes how concurrent applications can be rapidly deployed and managed through means of a web framework, which acts as a broker, carefully considering which applications need to be shown by use of a web browser. Other work [1] has led to the development of bespoke applications which launch on individual devices to display specific content, which has been demonstrably shown to lack openness and scalability. This approach is largely due to the fact that most public displays are proprietary technology, in which the network acts as an "isolated island, each with its own concepts and technologies". This is both a problem for users and developers alike, with the latter requiring huge 
Figure 2. An overview of our open pervasive display ecosystem..

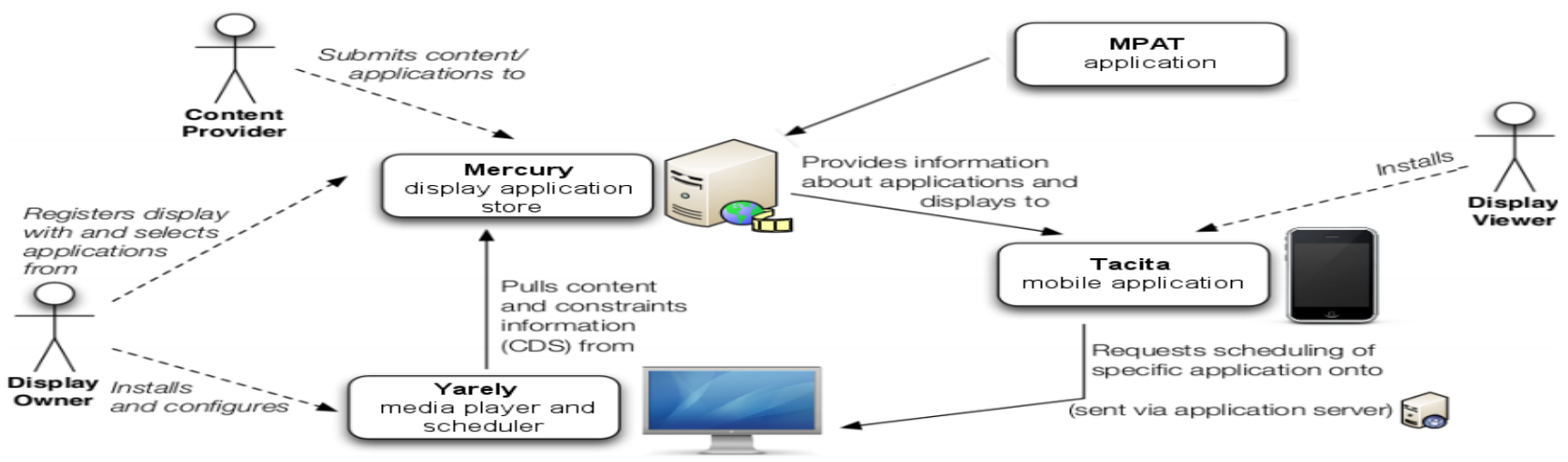

amounts of effort to build the foundations of the infrastructure and the interaction mechanism, within the limitations of the architecture. For the users, the bespoke nature of these infrastructures precipitates unfamiliarity with both identifying a public display and also the interaction model.

Due to the lack of abstraction within the sphere of public displays, the infrastructure adopted by Lancaster University is itself bespoke, however it offers abstraction in terms of application development by allowing 3rd parties access to a RESTful API which in turn aids the development process. Figure 2 depicts the ecosystem of the architecture and the interaction mechanism contained within. The MPAT application developed resides within mercury's application store, and can be instantiated upon demand via integration of web servers which serve requests. As shown, Yarely [4], a software player for open pervasive display networks, dictates the scheduling of application to be launched on specific displays. This technology allows accessibility for a range of different media types, including video and pdf. The application server is responsible for providing Yarely with a CDS (Content Descriptor Set). This is described in [4] as "a method to provide a description of a set of content items to be played by the node, the circumstances in which they should be played, and the location of any required media". This CDS is in XML format, which allows for a range of implementations, as from a transmission perspective it is protocol agnostic. The CDS must be rendered and manipulated for each individual scheduling request, and provides a layer of abstraction for application developers, whom only have to consider adhering to the API standard. This architecture also allows for web applications to be deployed on the various displays situated around campus, by specifying the type and URL within the CDS. This integral functionality allows MPAT applications to be natively launched on the displays, as MPAT itself is web-based and pages within the application have their own URL. Furthermore, encompassing MPAT applications within a web framework allows integration for all other PDIs which allow applications from a web source. As the pilot is fundamentally providing a non-linear story to users who traverse campus, the application server developed must distinguish between individuals and enumerate how many displays they have visited, in order to create a CDS which contains a hyperlink to the MPAT application where the correct video is stored. This CDS is then sent to the infrastructure, where the request is scheduled and eventually displayed. Upon receipt of a content request, the display launches a WebKit instance, which is directed to URL specified in the CDS.

\section{Application Development}

An MPAT application is a set of pages that are distributed with a piece of broadcast content, which should have a single style, entry point (the URL specified by the Application Information Table), origin, and which is created from a single application model. Each page is composed of multiple predefined components, which are bundled with the MPAT editor. Using this editor, components can be placed in locations on the page at the developers will, which will eventually create the desired application. There are two fundamental stages that a developer must traverse in order to make an MPAT application, the first being the creation of a page model.

The page model, shown in Figure 3, acts as a template for the various pages within the application, and is achieved in a visual fashion. Designers of the application can drag and drop boxes to the desired position within the page, which represent the various components that the MPAT editor offers. This avenue of application development is advantageous in multiple ways, however, one of the main advantages is the fact that no code will need to be written in order for a new application to be developed. This saves both time and money for prospective businesses wishing to create interactive TV applications, as expertise is not needed in this instance.

Figure 3. Page model editor.

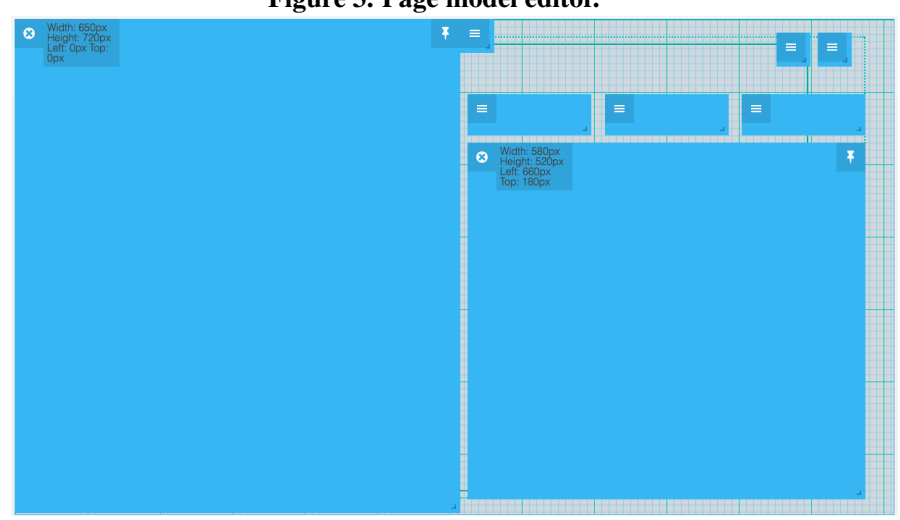


Figure 4. Component editor interface.

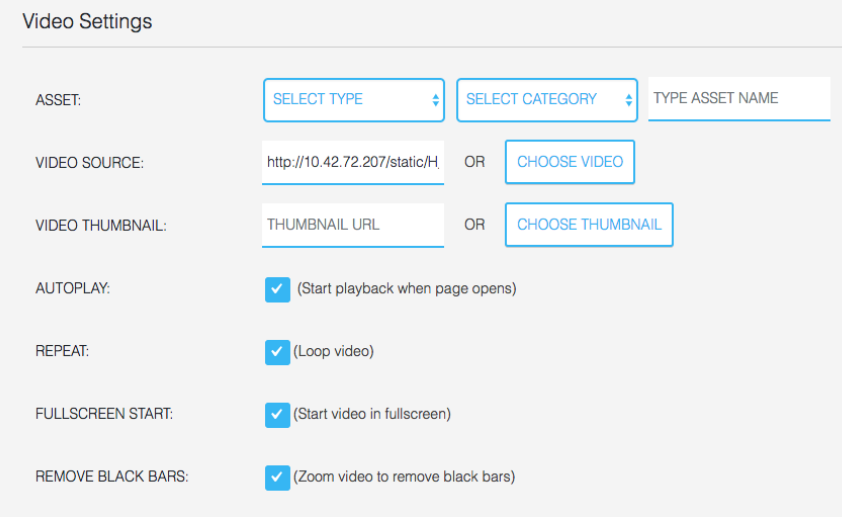

Once the overall layout page is satisfactory and suits the purpose of the application, the designer must then traverse the second stage of the authoring process, and navigate to the page editor within WordPress. The page editor requires a page model in order to build a template for the selected page. Once selected, components can be placed at will in the predefined positions set within the page model. Clicking on a components template within the editor will load the relevant component options menu, and will allow the user to specify which component is to be used and to customise the component at their will. For simple components such as text, the customisation options are limited and only provide basic support such as dictating the style and whether it can be navigable. However, more complex components such as video allow for a wider range of customisation ability, with the MPAT editor including optional functionality for autoplay, repetition, fullscreen start, and video thumbnails. Figure 4 illustrates the interface utilised by authors by customise the componenets.

This method of development not only vastly decreases the time and cost for the creation of interactive HbbTV applications, but also provides the functionality needed for applications within the sphere of public displays. As the digital prospectus pilot is a relatively complex application, with lots of different information needing to be displayed, the number of different components is vastly superior to the presence detection pilot. For the presence detection pilot, multiple pages within the same application were created to store the various videos, created by a digital artist, which make up the overarching storyline. These pages do not link to each other and have no method of navigation, and only contain a single video component.

Three different navigation models have been incorporated into the MPAT editor to create applications with different narrative approaches. The first is the website model, which migrates the link based navigation experience that the consumers are used with in mobile and computer web browsers; users will be able to navigate through the application and click on hyper links, using a remote control unit, to advance to the next stage. This method of navigation encompasses most non-specialised applications, and as it is in the same ilk as traditional web pages it is a popular choice among creators utilising the toolkit. Many varied types of applications have been created using this model, and allows creators much freedom in terms of design and navigational pathways.

Slideflow offers a more appealing story telling approach, where users can follow the narration scrolling in predefined directions. This approach assumes pages are located next to each other, and each page naturally leads to the next. This is achieved by implementation of HbbTV APIs, namely using the Key Events [3]. Hooks to the directional keys on the RCU (Remote Control Unit) are used by MPAT to ensure that they are only available to scroll through the application, and cannot be used by developers utilising the page editor.

Finally, the Timeline editor allow the developers to hook creative content, and therefore govern its appearance and behavior, to predefined events. This approach allows multiple pages to be displayed on the screen at any time, with the broadcast video present behind the application. This navigation scheme covers applications that are time oriented and is described in [16] as "either dependent upon media time in the case of applications such as VoD and catch-up, or on stream events in the case of live broadcasts where the precise synchronization of HbbTV elements is provided through stream events". One of the main driving factors for this style of navigation was for the vast popularity of 'red button' style applications. This style will periodically notify the existence of an application by means of an alert visible in front of the broadcast media. This is often used to indicate that additional context is available for consumption; whether that be the showcasing of contextual information relevant to current events within the broadcast content, such as social media integration, or some other form of entertainment medium that users can consume. Once the red button has been pressed, an MPAT application will be dynamically loaded onto the TV which will deliver the additional content.

Consideration of the type of application which needs to be created must be applied when deciding which navigation style is to be implemented. For some applications such as ones which make use of the "red button", this decision is fairly straightforward, however, for most this choice involves the weighing and evaluation of multiple aspects concerning the application architecture, requirements, and most importantly the setting in which the application will be deployed.

For the digital prospectus pilot, the Timeline navigation model was chosen. This was largely chosen due to it requiring ambient video, with notifications suggesting the presence of an application which provides more specific contextual information. This was the obvious choice, as this model was fundamentally developed for this style of application. For the presence detection pilot, a standard website model was chosen. Whilst any model would have fit this application, as the PDI simply creates a web request and displays a page from an unnavigable application, there is no need for implementation of the more heavyweight counterparts.

\section{IMPLEMENTATION}

As mentioned previously, the first use case focuses on building an electronic prospectus for students visiting Lancaster University on one of our open days. This study makes use 
of a looping video showing different aspects of the School and Computing and Communications, as well as red button functionality, which allows prospective students to find out more information about the content currently being displayed. The video being shown was situated in a room filled with staff members from individual departments within Lancaster University, with the intention that prospective students shall navigate to the department they are interested in, in order to communicate with staff to gain relevant knowledge. Naturally, with the number of students attending being many more than the amount of computing staff available at the even, students may have to wait a period of time until a staff member is available to talk to and gain the relevant information that they require. Due to this, the prospectus application was devised to give students, whom would be otherwise standing and waiting in line, an opportunity to find information about the University and the courses offered within, without direct contact with faculty members.

As this pilot did not utilise the existing public display infrastructure, the method of loading the application was more trivial, and required the use of BRAHMS [11], which is an industry standard transport stream multiplexer and provides the broadcast channel for the display to play. This also provides an easy way to load the MPAT application, which is done by entering a URL, which is then subsequently parsed into the DVB-T2 Application Information Table (AIT), which is a table holding relevant information needed to execute an application. This allows the HbbTV enabled display to load the application remotely, using a network connection.

The application created consists of two parts, firstly, the red button which takes an end user to the corresponding page within the electronic prospectus, and the electronic prospectus itself. An informative message is displayed at predefined time slots within the video which informs students that additional contextual information is available. A remote control unit was utilised by the students to initially gain access to the prospectus, when a notification of additional context appears upon the display, and then to navigate through the pages located within.

Figure 5. Notification of additional context.

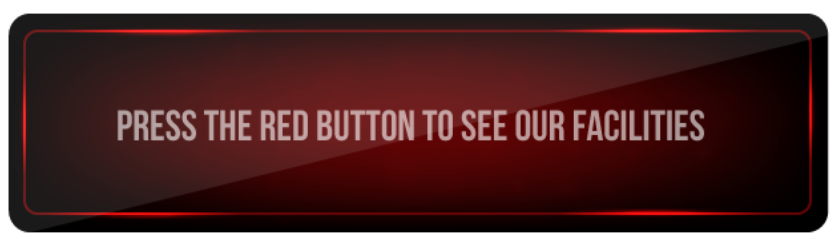

The standard functionality of this case study consists of looping video which contains footage of several aspects within Lancaster University and the School of Computing and Communications itself, including aerial footage of InfoLab21, a building housing researchers and other faculty members involved in the computing department, and research projects spearheaded by the various students within the department. Notifications of additional context are pushed to the display once relevant content within the video is shown, and if a user decides to press the red button during that period, MPAT will load the relevant page within the prospectus application. This is achieved by utilisation of the aforementioned Timeline feature. The Timeline editor enables applications to be visible in front of the looping video at specific periods of time.

Figure 5 shows an example banner which is pushed via time events within the Timeline editor. This banner appears within certain time periods of the broadcast video, and is set to predefined times within the editor. This banner loads the relevant application once a user presses the red button on the remote control unit. Based on an overview of the courses offered within the School of Computing and Communications, the prospectus offers the visitors a chance to gain additional information about subject areas they are interested in. The various pages contained within the application can be navigated by selecting the link components, which serve as pathways between content areas.

Grouping of related information is achieved by the aforementioned hotspot component. The different courses offered by the School of Computing \& Communications can be chosen within the disparate standalone pages. Selecting a hotspot component invokes a state change, which updates the page to show relevant information about the hotspot selected.

Figure 6. Implementation of digital prospectus.

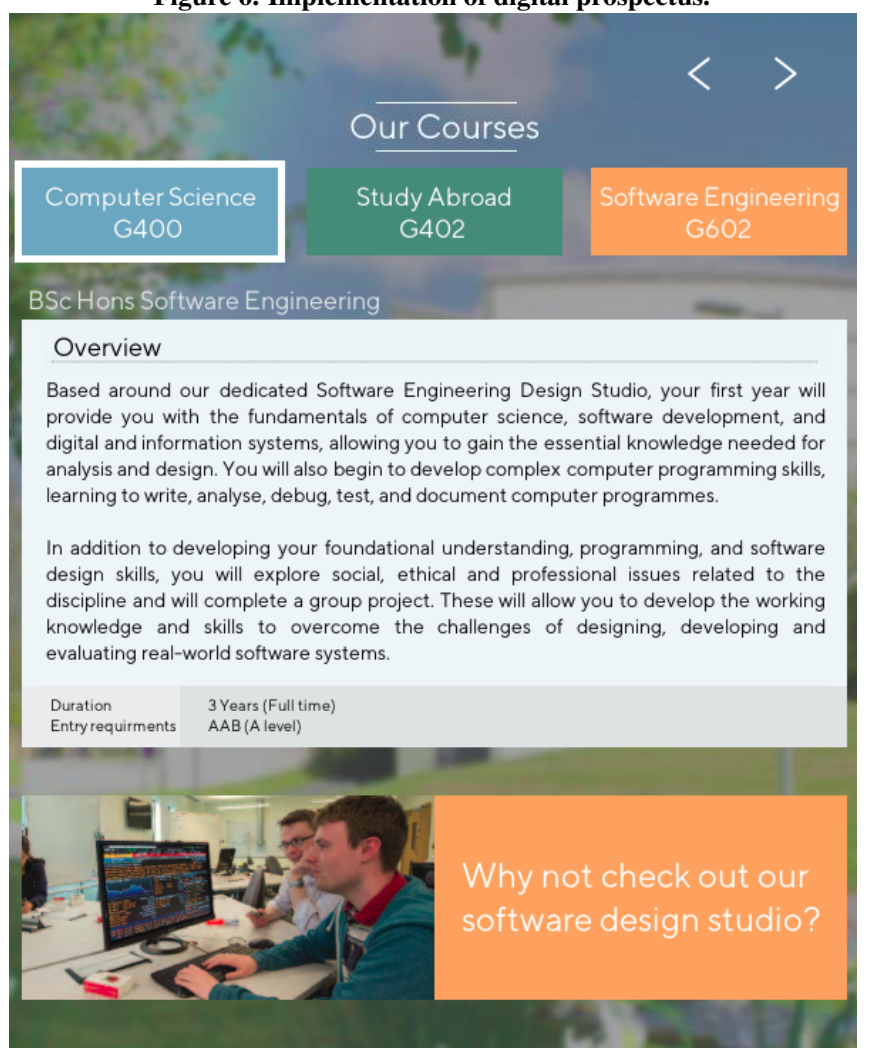

Figure 6 shows a page within the digital prospectus application created, and shows the functionality of the hotspots which have been implemented in the form of navigable boxes which represent a course available on the currently viewed page. 
Figure 7. Communication between entities.

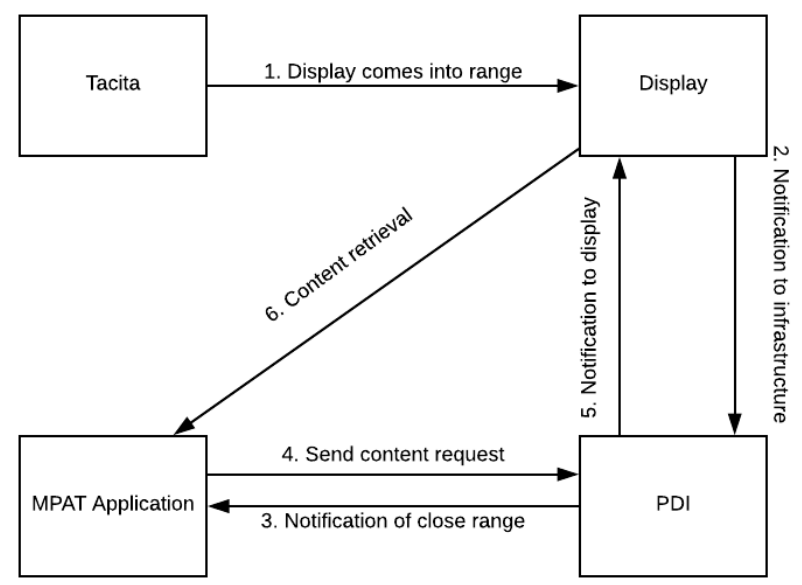

The presence detection pilot aims to demonstrate the possibilities of HbbTV outside of the traditional broadcast format, whilst also simultaneously promoting interactivity amongst collegiate and visitors around campus. This large scale pilot showcases content created by MPAT to a significant number of end-users.

The pilot relates to presence of users, and requires the use of Tacita's API to receive events from the iOS application, individual displays and the submission of content requests. In order to conform to the API standards Tacita upholds, a web server was created which accepts various requests from the PDIs RESTful interface. The architecture of the system, and the steps which need to be undertaken in order to display content on a display is outlined below in Figure 7.

The application server which was created, implemented a bespoke user management system which tracked the individuals route around campus, noting the displays which they passed along the way. Each time a request from a device with Tacita installed was received, a cookie was attached which uniquely identified the user. If this cookie had not been processed before, then a new user had initiated contact with the application server, and a new user object was created which also appended the display which triggered the request. This functionality enabled the application server to dictate which video that a user should receive.

Once a request for content is received, the application server first checks how many displays they have been to previously, and then which storyline group the display belongs to. Once this information has been retrieved, a response to the displays GET request is formulated. The response will be a 302 redirect to an external MPAT application which contains the correct video. Upon receipt of this response, the display will open a WebKit instance of the MPAT application, and the video will begin playback. This pilot showcases the capacity for MPAT to extend beyond the traditional TV broadcast medium, and provide an interactive storytelling narrative across heterogeneous devices.

\section{INTERACTION}

The digital prospectus pilot implemented the same method of interaction that is traditionally used in digital TV applications, by means of a remote control unit. While this could be seen as advantageous, as many users would be familiar with this style, there are also noticeable drawbacks. Many public displays are now touch screen or have some other method of input, and as a result, the general public may have difficulty correctly identifying that it is a TV displaying the broadcast, and that a remote is required. Several avenues were explored to remedy this, and have been discussed in the design section. While the interaction procedure may be verbose, the method of input can be seen as a simple task. The users of the application were able to correctly launch the initial application, and then intuitively navigate through the pages to gather the information that they require. The procedure required involves initially pressing the red button on the RCU, in order to launch the main functionality of the application. Once displayed, navigation through the pages is also achieved by using the RCU. Focus to different elements within the page is achieved by a white border around the piece of content, which indicates which element is currently selected. Standardised navigation between page elements has been implemented in such a way that keys can change the selected element by direction: clicking on the left arrow passes the focus to the nearest element to the left of the current focused zone. If there is none, nothing happens. Enter is used to trigger the action of the current focused zone, for example initiating playback of multimedia.

The interaction with the PDI is very different from the previous pilot and focuses on the combination of the Tacita application and RFID technology to detect the presence of subscribed users. Once installed, a list of sub-applications is visible from the user interface, in which the user can subscribe to. If a subscription to the application is enabled, once a user comes into close proximity to a display and it's corresponding iBeacon, the Mercury application store which houses the applications used by Tacita, receives a request for content, and the relevant video is displayed. This method of interaction is completely hands-free and requires little input from the user other than the initial installation of the application. Due to this minimal interaction procedure, users will not feel frustrated in attempting to learn new verbose and bespoke methods in order to make use of the functionality provided.

Figure 8. Still image of a part of the artist narrative.

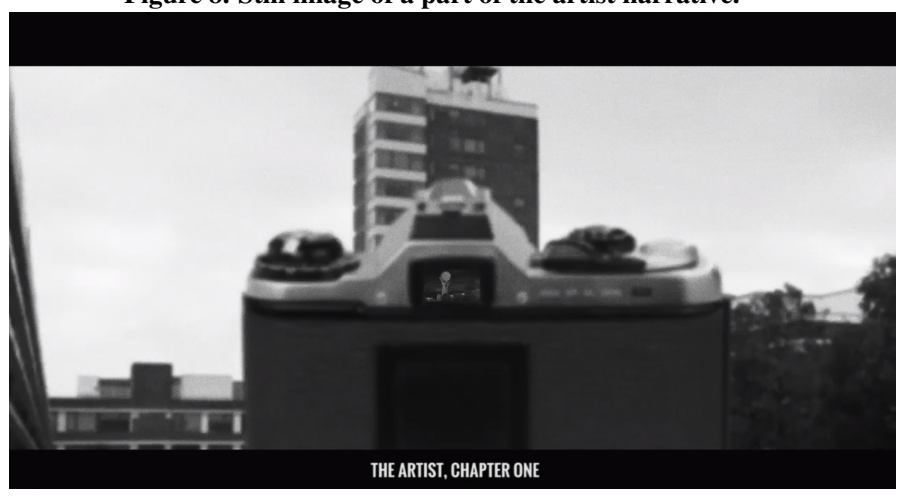


Figure 9. Utilisation of the digital prospectus application.

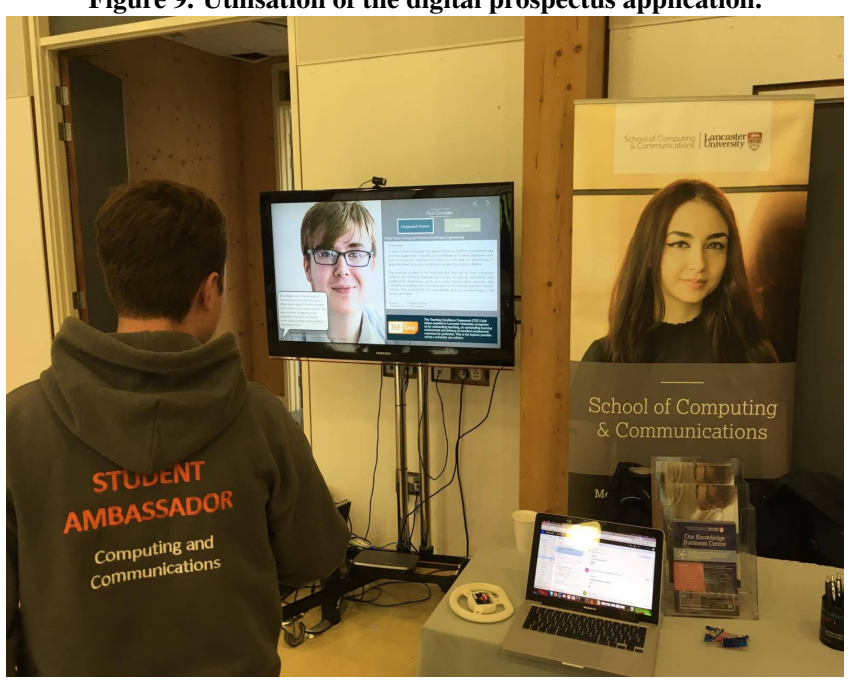

\section{EVALUATION}

\section{Digital Prospectus Pilot}

The digital prospectus pilot has been deployed in two separate instances. The pilot was active during two university open days and enabled prospective students to witness the capabilities of the digital authoring tool in a public setting. The display was situated at the fore of the respective departmental stalls, in an attempt to capture the attention of potential users. Large banner notifications were chosen as means to notify the existence of an application, and a remote control unit was placed in plain sight close to the relevant department's informational desk, with a large note stating that it can be used to interact with the display.

Figure 9 showcases the environment in which the application was deployed, and the setup of the display. Prospective students who were otherwise incapable of speaking directly to a member of staff would actively be drawn to the informational display, and correspondingly make use of the application. Other prospective students who would require information that is provided by the application would also be allocated time with the application after engaging in conversation with a member of staff. Users were receptive to the application and spent varying periods of time traversing the various pages held within, in order to find the information they require. Access $\operatorname{logs}$ of the system where the MPAT instance is installed were parsed, and subsequent requests for the various web pages within the application were accumulated.

\begin{tabular}{|l|c|c|r|}
\hline Page & Pilot 1 & Pilot 2 & Total \\
\hline Landing Page & 28 & 24 & 52 \\
\hline Undergraduate Courses & 19 & 19 & 38 \\
\hline Masters Courses & 17 & 12 & 29 \\
\hline Combined Degrees & 11 & 7 & 18 \\
\hline Final Year Project & 6 & 4 & 10 \\
\hline Our Facilities & 11 & 6 & 17 \\
\hline Total & 92 & 72 & 164 \\
\hline
\end{tabular}

As shown, the most popular pages were the landing pages at the start of the application. This indicates that many users did not fully traverse the application, and instead selected only content that was relevant to them. The number of users who interacted with the system can be said to be approximately equivalent to the number of hits to the landing page. However, as mentioned previously other pages can be launched into directly, depending upon the context currently within the ambient video. As such, the number of total users is likely in excess of the reported 52 .

The application development process was understandably a rapid experience, consisting of no more than 24 hours until completion of the application. A collaborative effort was made by the development team using the MPAT visual editor to concurrently create the web pages contained within using a single page model. The editor allows for cloning of pages and the components contained within, which grants separate entities the chance to easily reuse similar page styles for faster creation.

\section{User Evaluation}

A number of participants were involved in a user evaluation which aimed to gauge the overall satisfaction of the product. The usability of the application is evaluated in multiple aspects, which include questionnaires, specific tasks to undertake, and open-ended interview questions. While the questionnaire aims to gather both an understanding of the users background and the vital usability ratings, the interview questions additionally grants the accumulation of rich qualitative data. Ten people participated in the evaluation, all of whom are prospective students and between the ages of 18 to 25 , which were chosen to represent the population of potential users of the application. The number of users involved in the evaluation contained more males (7) than females (3).

The evaluation was conducted over a 2 day period, with each day having a fraction of the participants interacting with the study. Participants were first given written instruction on how to operate the application, and then encouraged to become familiar with the process. They were then instructed to perform tasks such as finding a piece of information from the prospectus. All of the participants were successful in navigating through the application and correctly identifying the required information. Participants were then asked to fill out a questionnaire, which included a 7-point Likert type scale to examine specific usability metrics. A subset of the specific user satisfaction questions are listed below:

Q1 The application positively affects my view of the University.

Q2 The application allows me to find information I am interested in.

Q3 The application gives me a better understanding of the University.

Six participants $(60 \%)$ reported a rating of $\geq 5$ when asked if the application positively affects their view of the University, while only one $(10 \%)$ reported a rating of $\leq 3$. In relation to whether the application allows the participants to find information they are interested in, eight $(80 \%)$ declared a score of $\geq 5$, and no participants gave a rating of $\leq 3$. Seven par- 
ticipants (70\%) gave a rating of $\geq 5$ when asked whether the application grants a better understanding of the University, while one (10\%) gave a rating of $\leq 3$. All of the participants also declared that they were familiar with public displays.

During the interview stage of the evaluation, a common comment was that having the ability to find additional information about the building they were located inside, was valuable in providing contextual information which was previously unknown to them. Furthermore, a large portion of participants described that gaining this information without the need to communicate with members of staff was beneficial. The participants who gave a rating of $\geq 5$ for $\mathrm{Q} 1$, listed above, were asked to elaborate on the reason why they gave that response. A prevalent opinion by the participants was that they were excited to see useful products which benefit student wellbeing being spearheaded by the University. The qualitative evaluation also highlighted some areas where confusion may arise for participants. The landing page, which was used to gauge the amount of users interacting with the application, was seen by many users as being obtuse. The landing page consisted of a splash screen with the MPAT logo and a button which the user must press in order to continue to the main content of the application. This piece of functionality was expressed to be unimportant when compared to the rest of the application. Moreover, the lack of an on screen explanation was said to detriment the understanding of the users.

Upon reflection of the usability evaluation, changes were made to the application which aim to accommodate the points of concern raised by the users. Amendments to the interface were included in a revised prototype, which include textual on screen instructions which aid the user navigate to the main functionality. It is recommended for future deployments to remove the landing page, and instead specify the entry point of the application to be the first page of the informational content.

\section{Presence Detection Pilot}

The presence detection pilot, which utilised personalisation and mobile sensing, was active for a month, and yielded many results due to active participation by collegiate members. As the 'iLancaster' application is a prerequisite for undergraduate students and thus installed on multiple devices, they had the option to use the external applications and engage with the pilot. The pilot made use of all 45 connected public displays situated around the University campus, and allowed students to create their own narrative dependent upon the route taken. During the period in which the application was live, 256 unique video requests were served by the PDI. This equates to over 50 possible unique narratives which have been explored by various faculty members.

The data collected from this pilot also grants insight into usage patterns, as a log of each display interaction is recorded inside a database. Analysis of the results indicate the vast majority of videos served by the PDI were between lecture periods. Furthermore, only a minute portion of video requests were served after lectures had finished for the day. This naturally leads to the conclusion that the students who were active in the pilot wanted to engage with it in between periods of learning when otherwise unoccupied. This notion is reinforced by the location of the displays accessed. The data collected reveals that a large portion of accessed displays were located within close proximity to Bowland College, the main hub for lecture theatres within Lancaster University.

The web based development framework has been shown to be a suitable approach for public display applications, as this method allows for multi-platform implementation. Furthermore, this aspect opens up possible integration with other PDIs which accept content from third-party sources through publication of services. Content creation via instantiation of functionality provided by MPAT has been shown to be successfully utilised, and engaged in by multiple members of the public, from various backgrounds, which have garnered both useful statistics and user experience metrics. The results gained coupled with the correct utilisation of the implemented MPAT applications suggest encouraging results and promising further deployment of newly created applications.

\section{FUTURE WORK}

The success of the digital prospectus application suggests similar MPAT application could be created and operated in other informational institutions which allow patrons to find contextual information without requiring communication with external entities. Interest has been garnered within the computing department to house the application permanently in InfoLab21, the building which houses computer science researchers and staff, as a tool for visitors outside of open day events. A proposed solution is to install a display in the foyer, which is constantly executing the MPAT application, in an attempt to attract individuals for interaction purposes, whilst also gaining ambiance for frequenters of the building.

This informative style of application allows for multiple avenues to be explored upon examination of suitable environments. Suitable venues could include, but are not limited to, recreational facilities with an emphasis on learning such as aquariums or museums. It is proposed that these institutions create applications for individual exhibits, which will allow patrons the opportunity to find contextual information about the pieces they are currently viewing in an interactive manner. Due to the ease of use that MPAT provides, creation of said applications will not require large up front costs for technicians with expertise, whilst also providing a comprehensive learning experience for visitors.

The ability to create public display applications in an efficient manner by leveraging the functionality which MPAT provides paves the way for organisations to utilise the open source tool and implement their own infrastructure. Due to the lack of abstraction inherent within PDIs, standardisation of technology which allows rapid and multi-platform deployment would be truly beneficial. This abstraction layer provides fundamental changes in application development life cycle and intrinsically depreciates the effort required to create complex interactive public display applications, while also maximising possible compatibility across devices. This compatibility could lead to future implementation of MPAT applications tailored towards integration with other PDIs that support web based applications. 


\section{CONCLUSION}

This paper presents the design, implementation and evaluation of MPAT applications catered towards instantiation upon public displays. Several challenges have been identified through research into the problem space, and through real world case studies these have been mitigated through correct utilisation of application design and interaction procedure. Creation of public display applications from the visual authoring environment that MPAT provides has been demonstrably shown to be comparable to other much more bespoke alternatives, while greatly diminishing the deployment cost.

\section{ACKNOWLEDGMENTS}

This work was supported by the Collaborative Project MultiPlatform Application Toolkit (www.mpat.eu) funded by the European Commission through the Horizon 2020 Programme (H2020-ICT-2015, call ICT19-2015) under grant agreement number 687921 .

\section{REFERENCES}

1. Jorge C. S. Cardoso and Rui Jose. Interaction Tasks and Controls for Public Display Applications. In Advances in Human-Computer Interaction. 2014. DOI=10.1155/2014/371867

2. Tomas Linden, Tommi Heikkinen, Timo Ojala, Hannu Kukka, and Marko Jurmu. Web-based framework for spatiotemporal screen real estate management of interactive public displays. In Proceedings of the 19th international conference on World wide web. 2010. DOI=http://dx.doi.org/10.1145/1772690.1772901

3. HbbTV Specification 2.0 https://www.hbbtv.org/ wp-content/uploads/2015/07/HbbTV_specification_2_0.pdf

4. Sarah Clinch, Nigel Davies, Adrian Friday and Graham Clinch. Yarely : a software player for open pervasive display networks. In PerDis' 13 Proceedings of the 2nd ACM International Symposium on Pervasive Displays. 2013.

5. Thomas Kubitza, Sarah Clinch, Nigel Davies, and Marc Langheinrich. Using mobile devices to personalize pervasive displays. In SIGMOBILE Mob. Comput. Commun. 2013. DOI=http://dx.doi.org/10.1145/2436196.2436211

6. Market share trends for content management systems for websites https://w3techs.com/technologies/history_ overview/content_management

7. Alessandro Bendinelli and Fabio Paterno. Authoring Public Display Web Applications: Guidelines, Design Patterns, and Tool Support. In Proceedings of the 11th Biannual Conference on Italian SIGCHI Chapter. 2015. DOI=http://dx.doi.org/10.1145/2808435.2808457

8. Carlos Antonio Navarrete Puentes and Jose Tiberio Hernandez Penaloza. HEd: A Flexible HbbTV WYSIWYG Visual Authoring Tool. In Adjunct Publication of the 2017 ACM International Conference on Interactive Experiences for TV and Online Video. 2017. DOI=https://doi.org/10.1145/3084289.3089917
9. Tomas Linden, Tommi Heikkinen, Vassilis Kostakos, Denzil Ferreira, and Timo Ojala. Towards multi-application public interactive displays. In Proceedings of the 2012 International Symposium on Pervasive Displays. 2012. DOI=http://dx.doi.org/10.1145/2307798.2307807

10. Jorge C. S. Cardoso and Rui Jose. Creating web-based interactive public display applications with the PuReWidgets toolkit. In Proceedings of the 11th International Conference on Mobile and Ubiquitous Multimedia. 2012. DOI=http://dx.doi.org/10.1145/2406367.2406434

11. BRAHMS https://www.irt.de/en/products/ media-services-and-applications/brahms/

12. Tacita https: //itunes . apple. com/gb/app/tacita/id1131274089?mt=8

13. MPAT. http://www.mpat.eu/.

14. Sarah Clinch, Nigel Davies, Adrian Friday, Miriam Greis, Marc Langheinrich, Mateusz Mikusz, Thomas Kubitza and Christopher Winstanley. Demo : an ecosystem for open display networks. In PerDis '14 Proceedings of The International Symposium on Pervasive Displays. 2014.

15. MPAT Initial Plan for the Dissemination and Exploitation of Results https://ec. europa.eu/research/participants/documents/ downloadPublic?documentIds=080166e5ae913e09\&appId=PPGMS

16. MPAT System Architecture and API Documentation https://ec. europa.eu/research/participants/documents/ downloadPublic?documentIds $=080166 \mathrm{e} 5 \mathrm{abb} 3 \mathrm{cc} 63 \&$ appId=PPGMS 